\section{Predictive factors and outcomes of posterior segment intraocular foreign bodies}

\author{
Abstract \\ Purpose To identify the predictive factors for \\ anatomical and visual outcomes in posterior \\ segment intraocular foreign body (IOFB) \\ patients managed by pars plana vitrectomy \\ (PPV).
}

Methods A retrospective chart review was performed for 77 patients, who had PPV for IOFBs removal between January 2001 and July 2007. Univariate and multivariate analyses were conducted to evaluate the predictive factors. Results The mean age of the patients was 33.4 years. The nature of IOFBs was mainly metal, with injuries most commonly caused by an electric grass trimmer. The mean time interval between injury and IOFBs removal was 30.7 days. The mean preoperative visual acuity (VA) was 1.82 logarithm of the minimal angle of resolution (logMAR), and mean final was VA 1.10 $\log$ MAR. From multivariate analysis, good visual outcome was correlated with the corneoscleral entry site, and poor visual outcome was correlated with the

Department of Ophthalmology, Faculty of Medicine, Chiang Mai University, Chiang Mai, Thailand

Correspondence: J Choovuthayakorn, Department of Ophthalmology, Faculty of Medicine, Chiang Mai University, 110 Intawaroros Road, Chiang Mai 50200, Thailand

Tel: + 6653 9455123;

Fax: + 6653946121

E-mail: janjitc@yahoo.com

Received: 22 December 2010

Accepted in revised form: 28 July 2011

Published online:

16 September 2011 presence of relative afferent pupillary defect.

Conclusions In noncombat IOFBs, most cases were work-related. Delayed IOFBs removal was acceptable when primary wounds were repaired appropriately and extensive antibiotics administered. Even though advancement in vitreoretinal surgery can reduce the frequency of blindness, further promotion and education on eye protection during work are needed. Eye (2011) 25, 1622-1626; doi:10.1038/eye.2011.229; published online 16 September 2011

Keywords: intraocular foreign body; predictive factors; pars plana vitrectomy

\section{Introduction}

Open globe injury is an important cause of visual morbidity, especially in teenagers or young adults. Among open globe injuries
J Choovuthayakorn, L Hansapinyo, N Ittipunkul, $\mathrm{D}$ Patikulsila and $\mathrm{P}$ Kunavisarut

classified by Birmingham Eye Trauma Terminology, ${ }^{1}$ injury connected with sharp penetration had a better visual prognosis than that associated with blunt penetration, ${ }^{2}$ and intraocular foreign bodies (IOFBs) were reported as having better visual outcome when compared with other mechanisms. ${ }^{1}$ Prognosis for vision in IOFBs was dependent on confluent predictive factors, as published by previous authors. ${ }^{3-5}$ Because of the advancement in preoperative care and microsurgical technique during this era, several severely traumatized ophthalmic conditions can be managed successfully. The objectives of this study were to identify the predictive factors and evaluate the visual outcomes in posterior segment IOFB patients managed by pars plana vitrectomy (PPV).

\section{Patients and methods}

The patients, diagnosed as having IOFBs at Chiang Mai University Hospital between January 2001 and July 2007, were identified. Those with anterior segment IOFBs, enucleation as the primary treatment, and a postoperative follow-up period of $<1$ month were excluded from the study. The medical records of the remaining patients with posterior segment IOFBs, who underwent PPV, were reviewed retrospectively. The data collected comprised age, gender, mechanism of injury, preoperative visual acuity (VA), initial ocular features, nature of foreign bodies (FBs), time of interval from injury to IOFBs removal, postoperative VA, and complications. The VA was measured with a standard Snellen acuity chart and converted to a logarithm of the minimal angle of resolution $\left(\log\right.$ MAR) units for statistical analysis. ${ }^{6}$

All patients with a leaking wound underwent primary globe repair, performed by general ophthalmologists, before consultation with the retina unit for IOFBs removal. Patients with a self-sealing wound had PPV carried out as 
initial intervention. A standard three-port, 20-gauge vitrectomy technique was performed by four retinal specialists (NI, DP, JC, and PK). Lensectomy or phacoemulsification was also done in cases of traumatic cataract or lens injury. FBs were extracted through enlarged sclerotomy or limbal incision using intraocular forceps with or without the assistance of an external magnet. Any injured retinal areas, including detached retina, were secured by endolaser photocoagulation, cryoretinopexy, and intraocular tamponade by either gas or silicone oil. During the follow-up period, a good visual outcome was determined as postoperative final VA equal to or better than $20 / 40$, and a poor visual outcome as final VA of less than 20/200. Anatomical success was defined as no retinal detachment (RD) involving the macular area at the time of the last follow-up.

The predictive factors for endophthalmitis, anatomical success, and visual outcomes were studied using univariate analysis (Fisher exact test, or Mann-Whitney $U$-test), and multivariate logistic regression analysis. This study was approved by the research ethics committee of the Faculty of Medicine, Chiang Mai University, Chiang Mai, Thailand.

\section{Results}

Of the 84 patients diagnosed with IOFBs, 7 were excluded from the study, 1 because of an anterior segment FB, 4 because of primary enucleation, and 2 with a postoperative follow-up time of $<1$ month. The remaining records of 77 pairs of eyes (77 patients) were included in this study, with a mean follow-up time of 10.7 months (range 6-75, median 5.5, SD 14.3 months), and mean age of 33.4 years (range 7-70, median 34, SD 13.8 years). Seventy $(70 / 77,90.9 \%)$ patients were male. In all, $48(48 / 77,62.3 \%)$ patients had injury in the right eye and $29(29 / 77,37.7 \%)$ in the left one. The nature of the FBs was metal in $37(37 / 77,48.1 \%)$ patients, soilcontaminated object in $21(21 / 77,27.2 \%)$, gunpowder in 7 $(7 / 77,9.1 \%)$, wood in $5(5 / 77,6.5 \%)$, rock in $4(4 / 77$, $5.2 \%)$, and glass in $3(3 / 77,3.9 \%)$. The mechanisms of injury are summarized in Table 1 . Of these, 53 (53/77, $68.9 \%$ ) patients had work-related injury. The mean preoperative VA was 1.82 (range 0.00-3.00, median 0.78, SD 0.75) $\log$ MAR. The preoperative clinical data of the patients are presented in Table 2. The retinal injuries, comprising retinal hemorrhage, retinal break, and RD, occurred in $54(54 / 77,70.1 \%)$ patients.

Intravenous gentamicin and cefazolin were administered systemically during the preoperative period in $58(58 / 77,75.3 \%)$ patients. Nineteen (19/77, $24.7 \%$ ) patients, with a long-standing undiagnosed IOFBs or self-sealing wound and no clinical signs of infection, received no preoperative systemic antibiotics. The overall
(77 patients) mean duration between injury and IOFBs removal was 30.7 days (range 0-347, median 6, SD 71.25 days), whereas in the group receiving no preoperative antibiotics was 127.6 days (range 11-347, median 96.5, SD 113.8 days). Endophthalmitis developed in seven $(7 / 77,9.1 \%)$ patients; three from soil-contaminated object, three from metallic FBs, and one from rock. In clarifying the nature of IOFBs, 1 of $4(25.0 \%)$ among rock, 3 of $21(14.3 \%)$ among soil-contaminated object, and 3 of $37(8.1 \%)$ among metallic IOFBs were associated with endophthalmitis. The nature of FB showed no statistical correlation to endophthalmitis development (Fisher exact test, $P=0.322,0.383$, and 1.000 for rock, soil-contaminated object, and metal, respectively). In the endophthalmitis group, the mean interval from injury to IOFBs removal was 6.6 days (range 2-12, median 7, SD 3.74 days), and six of seven $(85.7 \%$ ) patients received preoperative systemic antibiotics. A delayed surgery of more than 7 days (Fisher exact test, $P=0.430$ ) and preoperative antibiotics (Fisher exact test, $P=0.674$ ) showed no statistical correlation with endophthalmitis development. Despite having signs of infectious

Table 1 Mechanisms of injury

\begin{tabular}{lc}
\hline Mechanism of injury & Number (\%) \\
\hline Electric grass trimmer used & $25(32.5)$ \\
Chiseling on metal & $13(16.9)$ \\
Hammering on nail & $10(13.0)$ \\
Firework explosion & $8(10.4)$ \\
Grenade explosion & $7(9.0)$ \\
Gun shot & $6(7.8)$ \\
Wood cutting & $5(6.5)$ \\
Car accident & $3(3.9)$ \\
\hline
\end{tabular}

Table 2 Clinical data of the patients

\begin{tabular}{lc}
\hline Clinical characteristics & Number (\%) \\
\hline Penetrating site & \\
$\quad$ Cornea & $61(79.2)$ \\
Sclera & $10(13.0)$ \\
Corneosclera & $6(7.8)$ \\
RAPD & $18(23.4)$ \\
Hyphema & $32(41.6)$ \\
Iris injury & $41(53.2)$ \\
Lens injury & $66(85.7)$ \\
Vitreous hemorrhage & $55(71.4)$ \\
& \\
Retinal injury & \\
$\quad$ Retinal break & $28(36.4)$ \\
Retinal hemorrhage & $21(27.3)$ \\
Retinal detachment & $5(6.5)$ \\
Endophthalmitis & $7(9.1)$ \\
Siderosis bulbi & $3(3.9)$ \\
\hline
\end{tabular}

Abbreviation: RAPD, relative afferent pupillary defect. 
endophthalmitis such as hypopyon, gram stain identified Gram-positive cocci in only three eyes and cultures were negative in all seven cases. All patients with preoperative endophthalmitis had a final VA of less than $6 / 60$. None of the predictors, including nature of FBs, preoperative antibiotics, or interval before IOFBs removal, were found to be associated significantly with endophthalmitis by univariate analysis.

Siderosis bulbi was seen in $3(3 / 77,3.9 \%)$ metallic IOFBs patients. In each of two patients, the duration before vitrectomy was 283 and 312 days, respectively, but in one patient, the history of trauma was undetermined. The final VA was equal to or better than $20 / 200$ in two $(2 / 3,66.7 \%)$ patients, and worse than $20 / 200$ in one $(1 / 3,33.3 \%)$. Siderosis bulbi did not show a statistical association for final visual outcome (Fisher exact test, $P=1.000)$.

Lens removal was performed at the time of vitrectomy in $55(55 / 77,71.4 \%)$ patients. Intraocular tamponade was performed at the end of surgery in $25(25 / 77,32.5 \%)$ patients. Of these, $12(12 / 77,15.6 \%)$ were silicone oil, and $13(13 / 77,16.9 \%)$ gas tamponade. At the end of follow-up, six $(6 / 77,7.8 \%)$ patients had failed to achieve anatomical success; three $(3 / 77,3.9 \%)$ from severe proliferative vitreoretinopathy, two $(2 / 77,2.6 \%)$ from phthisis bulbi, and one $(1 / 77,1.3 \%)$ from surgery rejection by the patient. Anatomical success was achieved in $71(71 / 77,92.2 \%)$ patients. The most common complication of anatomical failure was PVR. Intraocular silicone oil tamponade, intraocular gas tamponade, or time of interval before IOFBs removal of equal to or less than 7 days did not show a statistical correlation for final anatomical success (Fisher exact test, $P=0.266,0.234$, or 0.213 , respectively). Absence of initial RD demonstrated as a significant associated predictor for anatomical success by univariate analysis (Fisher exact test, $P=0.00$ ), but not with multiple logistic regression analysis.

The mean final VA was 1.10 (range 0.00-3.00, median 0.78 , SD 0.99) $\log$ MAR. Seven $(7 / 77,9.1 \%)$ patients reported no light perception after IOFBs removal. The final VA was improved in $49(49 / 77,63.60 \%)$ patients, stabilized in $15(15 / 77,19.50 \%)$, and worse in $13(13 / 77$, $16.90 \%)$. Among the patients with a preoperative VA of less than 20/200, $34(34 / 65,52.3 \%)$ had a final VA of equal to or better than $20 / 80$. Preoperative and final VAs are demonstrated in Table 3.

For good visual outcome, univariate analysis showed that absence of relative afferent pupillary defect, corneoscleral entry site, and absence of endophthalmitis (Fisher exact test, $P=0.012,0.022$, and 0.044) were the significant associated predictors (Table 4). Multivariate logistic regression analysis revealed that the corneoscleral entry site $(P=0.018$; odds ratio $=18.36$; $95 \%$ CI $1.63-206.10$ ) was a strongly associated predictive factor. For poor visual outcome, univariate analysis showed that presence of endophthalmitis, relative afferent pupillary defect, and initial RD (Fisher exact test, $P=0.002$, 0.006 , and 0.012 ) were the significant associated predictors (Table 5). Multivariate logistic regression analysis revealed that the presence of relative afferent pupillary defect $(P=0.001$; odds ratio $=8.58 ; 95 \%$ CI $2.42-30.40)$ was a strongly associated predictive factor.

\section{Discussion}

In either the noncombat or combat situation, IOFBs is a serious problem in a young, working age population. In accordance with previous reports, ${ }^{4,7-9}$ this study showed that the majority of patients $(90 \%)$ were male, with a mean age of 33.4 years. In the noncombat area, the incidence of IOFBs was significantly higher in the workplace than in the community. ${ }^{10}$ This study found that $68.9 \%$ of the patients had work-related injury.

Metal, obviously associated with hammering or

Table 3 Preoperative and final visual acuity

\begin{tabular}{lcc}
\hline$V A$ & $\begin{array}{c}\text { Preoperative VA } \\
\mathrm{N}(\%)\end{array}$ & $\begin{array}{c}\text { Final VA } \\
\mathrm{N}(\%)\end{array}$ \\
\hline$\geq 20 / 40$ & $6(7.8)$ & $28(36.4)$ \\
$<20 / 40-20 / 200$ & $6(7.8)$ & $12(15.6)$ \\
$<20 / 200$ & $65(84.4)$ & $37(48.0)$ \\
Total & $77(100)$ & $77(100)$ \\
\hline
\end{tabular}

Abbreviation: VA, visual acuity.

Table 4 Univariate analysis: predictors for good visual outcome

\begin{tabular}{lccc}
\hline Predictive factors & $\begin{array}{c}\text { Final VA } \\
\geq 20 / 40 \\
\mathrm{~N}(\%)\end{array}$ & $\begin{array}{c}\text { Final VA } \\
<20 / 40 \\
\mathrm{~N}(\%)\end{array}$ & P-value \\
\hline Mean age (years) & 29.5 & 32.5 & $0.624^{\mathrm{a}}$ \\
Sex (male: female) & $5: 1$ & $65: 6$ & 0.287 \\
Initial VA $\geq 20 / 40$ & $4(66.67)$ & $2(33.33)$ & 0.182 \\
& & & \\
Entry site & $22(36.07)$ & $39(63.93)$ & 1.000 \\
$\quad$ Cornea & $1(10.00)$ & $9(90.00)$ & 0.083 \\
$\quad$ Sclera & $5(83.33)$ & $1(16.67)$ & 0.022 \\
$\quad$ Corneosclera & $2(11.11)$ & $16(88.98)$ & 0.012 \\
RAPD & $21(31.82)$ & $45(68.18)$ & 0.194 \\
Lens injury & & & \\
Retinal injury & $7(25.00)$ & $21(75.00)$ & 0.223 \\
$\quad$ Break & $0(0.00)$ & $5(100.00)$ & 0.152 \\
$\quad$ RD & $6(28.57)$ & $15(71.43)$ & 0.439 \\
$\quad$ Hemorrhage & $0(0.00)$ & $7(100.00)$ & 0.044 \\
Endophthalmitis &
\end{tabular}

Abbreviations: RAPD, relative afferent pupillary defect; RD, rhegmatogenous retinal detachment; VA, visual acuity.

${ }^{a}$ Mann-Whitney $U$-test; in others Fisher exact test was used. 
Table 5 Univariate analysis: predictors for poor visual outcome

\begin{tabular}{lccc}
\hline Predictive factors & $\begin{array}{c}\text { Final VA<20/200 } \\
\mathrm{N}(\%)\end{array}$ & $\begin{array}{c}\text { Final VA } \geq 20 / 200 \\
\mathrm{~N}(\%)\end{array}$ & P-value \\
\hline Mean age (years) & 32.3 & 38.1 & $0.862^{\mathrm{a}}$ \\
Sex (male: female) & $61: 4$ & $9: 3$ & 0.084 \\
Initial VA $<20 / 200$ & $37(56.92)$ & $28(43.08)$ & 0.121 \\
& & & \\
Entry site & & & \\
$\quad$ Cornea & $26(42.62)$ & $35(57.38)$ & 1.000 \\
$\quad$ Sclera & $6(60.00)$ & $4(40.00)$ & 0.311 \\
$\quad$ Corneosclera & $1(16.67)$ & $5(83.33)$ & 0.230 \\
RAPD & $13(72.22)$ & $5(27.78)$ & 0.006 \\
Lens injury & $30(45.45)$ & $36(54.54)$ & 0.335 \\
& & & \\
Retinal injury & & $15(53.57)$ & 0.485 \\
$\quad$ Break & $5(46.43)$ & $0(0.00)$ & 0.012 \\
RD & $8(38.10)$ & $13(61.90)$ & 0.786 \\
$\quad$ Hemorrhage & $7(100.00)$ & $0(0.00)$ & 0.002 \\
Endophthalmitis & &
\end{tabular}

Abbreviations: RAPD, relative afferent pupillary defect; RD, rhegmatogenous retinal detachment; VA, visual acuity.

${ }^{a}$ Mann-Whitney $U$-test; in others Fisher exact test was used

chiseling, was the most common nature of IOFBs, which is similar to other studies. ${ }^{11-13}$ Interestingly, this study also revealed that the electric grass trimmer was the most common mechanism of injury.

Poor presenting VA has previously been reported as an important predictive factor for poor visual outcomes. ${ }^{7}$ However, our study showed no statistical correlation between preoperative VA of less than 20/200 and poor final VA (Fisher exact test; $P=0.121$ ). This may be due to improvement in medical and surgical care where many damaged structures; including cornea, lens, or retina, can be treated successfully. In the series of this study, 52.3\% of the patients, who presented with a VA of less than $20 / 200$, had a final VA of equal to or better than $20 / 80$.

It has been proposed that the risk of endophthalmitis decreases with early IOFBs removal. ${ }^{8,14,15}$ Other authors have demonstrated that delayed IOFBs removal was also appropriate when a primary wound was repaired promptly, with broad spectrum antibiotic administration $^{16,17}$ and no increased risk of endophthalmitis. As our medical center is a tertiary referral center, the mean time of IOFBs removal in this study was 30.7 days (median 6 days), whereas the incidence of endophthalmitis was $9.1 \%$, which is comparable to other studies; range 0-20\%. ${ }^{11-13,16}$

In a preceding report, certain different physical characteristics of magnetic and nonmagnetic IOFBs were associated with a higher rate of retinal break formation and subsequent RD, especially with glass IOFBs. ${ }^{18}$ This study did not demonstrate a correlation between the nature of IOFBs and postoperative RD (Fisher exact test, $P=1.000$ ). This might be because the numbers of glass
IOFBs in our series were small. A recent study reported an association of late onset RD and the separation of posterior hyaloids. ${ }^{19}$ None of the patients in this study had late onset RD.

A wide range of final visual outcomes was reported in the literature, due to the variability of causes. Good final visual outcome (VA of 20/40 or better) was reported in 30 to $71 \%$ of patients. ${ }^{5,7,20}$ In this study, $36.4 \%$ of the patients had good final visual outcome, which demonstrated statistically significant correlation with the corneoscleral entry site $(\mathrm{OR}=18.36)$. The corneoscleral entry site indirectly reflected absence of retinal injury, which damaged the visual pathway, and absence of corneal scar that precluded good visual outcome. Poor final visual outcome (VA of less than 20/200) was reported previously in 17 to $50 \%$ of patients, ${ }^{5,7}$ and in this study, $48.0 \%$ of the patients had poor final visual outcome, which demonstrated statistically significant correlation with the presence of relative afferent pupillary defect. However, the VA improvement observed within the first year after IOFBs removal could deteriorate from secondary complications during the second and third year, with stabilization occurring after the third year. ${ }^{9}$ With a limited follow-up period in this study, the final visual outcomes might change in some cases in long-term follow-up.

Limitations of this study were a retrospective nature, incomplete and varied data documentation, small number of patients, and limited follow-up period.

\section{Conclusions}

This study showed the difference between a nonindustrial and industrial penetrating eye injuries with IOFBs, in which most cases were work related. The most common cause was grass trimming with a lawnmower. Injury mechanism from grass trimming is increasing in our region of northern Thailand each year. Few patients, who were involved in gardening work, realized the importance of eye protection. Furthermore, firework explosion during festivals was the fourth mechanism of concern. Eye protection during risky work situations needs further promotion and education.

\section{Summary}

\section{What was known before}

- Delayed intraocular foreign bodies (IOFBs) removal may increase the risk of endophthalmitis.

\section{What this study adds}

- Delayed IOFBs removal was accepted when primary wounds were repaired appropriately and extensive antibiotics administered. Gardening work and firework explosion were among the mechanisms of concern in nonindustrial community. 


\section{Conflict of interest}

The authors declare no conflict of interest.

\section{Acknowledgements}

We thank Kittika Kanjanarattanakorn and Rochana Phuackchantuck, staff members of the research administration unit, Faculty of Medicine, Chiang Mai University Hospital, for their statistical assistance.

\section{References}

1 Globocnik Petrovic M, Lumi X, Drnovsek Olup B. Prognostic factors in open eye injury managed with vitrectomy: retrospective study. Croat Med J 2004; 45(3): 299-303.

2 Warrasak S, Euswas A, Hongsakorn S. Posterior segment trauma: types of injuries, result of vitreo-retinal surgery and prophylactic broad encircling scleral buckle. J Med Assoc Thai 2005; 88(12): 1916-1930.

3 Szijártó Z, Gaál V, Kovács B, Kuhn F. Prognosis of penetrating eye injuries with posterior segment intraocular foreign body. Graefes Arch Clin Exp Ophthalmol 2008; 246(1): 161-165.

4 Wickham L, Xing W, Bunce C, Sullivan P. Outcomes of surgery for posterior segment intraocular foreign bodiesa retrospective review of 17 years of clinical experience. Graefes Arch Clin Exp Ophthalmol 2006; 244(12): 1620-1626.

5 Erakgun T, Egrilmez S. Prognostic factors in vitrectomy for posterior segment intraocular foreign bodies. J Trauma 2008; 64(4): 1034-1037.

6 Schulze-Bonsel K, Feltgen N, Burau H, Hansen L, Bach M. Visual acuities 'hand motion' and 'counting fingers' can be quantified with the freiburg visual acuity test. Invest Ophthalmol Vis Sci 2006; 47(3): 1236-1240.

7 Greven C, Engelbrecht N, Slusher M, Nagy S. Intraocular foreign bodies: management, prognostic factors, and visual outcomes. Ophthalmology 2000; 107(3): 608-612.

8 Jonas J, Knorr H, Budde W. Prognostic factors in ocular injuries caused by intraocular or retrobulbar foreign bodies. Ophthalmology 2000; 107(5): 823-828.

9 Szurman P, Roters S, Grisanti S, Aisenbrey S, Rohrbach JM, Warga $\mathrm{M}$ et al. Primary silicone oil tamponade in the management of severe intraocular foreign body injuries: an 8-year follow-up. Retina 2007; 27(3): 304-311.

10 Kanoff J, Turalba A, Andreoli M, Andreoli C. Characteristics and outcomes of work-related open globe injuries. Am J Ophthalmol 2010; 150(2): 265-269. e262.

11 Demircan N, Soylu M, Yagmur M, Akkaya H, Ozcan A, Varinli I. Pars plana vitrectomy in ocular injury with intraocular foreign body. J Trauma 2005; 59(5): 1216-1218.

12 Woodcock M, Scott R, Huntbach J, Kirkby G. Mass and shape as factors in intraocular foreign body injuries. Ophthalmology 2006; 113(12): 2262-2269.

13 Ehlers J, Kunimoto D, Ittoop S, Maguire J, Ho A, Regillo C. Metallic intraocular foreign bodies: characteristics, interventions, and prognostic factors for visual outcome and globe survival. Am J Ophthalmol 2008; 146(3): 427-433.

14 Soheilian M, Abolhasani A, Ahmadieh H, Azarmina M, Dehgan MH, Mashavekhi A et al. Management of magnetic intravitreal foreign bodies in 71 eyes. Ophthalmic Surg Lasers Imaging 2004; 35(5): 372-378.

15 Chaudhry I, Shamsi F, Al-Harthi E, Al-Theeb A, Elzaridi E, Riley F. Incidence and visual outcome of endophthalmitis associated with intraocular foreign bodies. Graefes Arch Clin Exp Ophthalmol 2008; 246(2): 181-186.

16 Thach A, Ward T, Dick JN, Bauman WC, Madigan Jr WP, Goff MJ et al. Intraocular foreign body injuries during Operation Iraqi Freedom. Ophthalmology 2005; 112(10): 1829-1833.

17 Colyer M, Weber E, Weichel E, Dick JS, Bower KS, Ward TP et al. Delayed intraocular foreign body removal without endophthalmitis during Operations Iraqi Freedom and Enduring Freedom. Ophthalmology 2007; 114(8): 1439-1447.

18 Soheilian M, Feghi M, Yazdani S, Anisian A, Ahmadieh H, Dehghan MH et al. Surgical management of non-metallic and non-magnetic metallic intraocular foreign bodies. Ophthalmic Surg Lasers Imaging 2005; 36(3): 189-196.

19 Weissgold D, Kaushal P. Late onset of rhegmatogenous retinal detachments after successful posterior segment intraocular foreign body removal. Br J Ophthalmol 2005; 89(3): 327-331.

20 Wani V, Al-Ajmi M, Thalib L, Azad RV, Abul M, Al-Ghanim $\mathrm{M}$ et al. Vitrectomy for posterior segment intraocular foreign bodies: visual results and prognostic factors. Retina 2003; 23(5): 654-660. 